\title{
"Because if I don't hold his hand then I might as well not be there": Experiences of Dutch and UK care home visiting during the COVID-19 pandemic
}

\author{
Clarissa Giebel, 1,2 Bram de Boer, ${ }^{3,4}$ Mark Gabbay, 1,2 Paul Marlow, ${ }^{2}$ \\ Annerieke Stoop, ${ }^{5}$ Debby Gerritsen, ${ }^{6}$ and Hilde Verbeek ${ }^{3,4}$ \\ ${ }^{1}$ Department of Primary Care $\mathbb{E}$ Mental Health, University of Liverpool, Liverpool, UK \\ ${ }^{2}$ NIHR ARC NWC, Liverpool, UK \\ ${ }^{3}$ Maastricht University, Care and Public Health Research Institute, Department of Health Services Research, Maastricht, The Netherlands \\ ${ }^{4}$ Living Lab in Ageing and Long-Term Care, Maastricht, the Netherlands \\ ${ }^{5}$ Tranzo Department, Tilburg School of Social and Behavioral Sciences, Tilburg University, Tilburg, the Netherlands \\ ${ }^{6}$ Radboud University Medical Center, Radboud Institute for Health Sciences, Radboudumc Alzheimer Center, Department of Primary and Community Care, \\ Nijmegen, the Netherlands
}

Abstract

Objectives: To explore and compare the experiences of care home visits during the pandemic in the UK and the Netherlands.

Design: Qualitative semi-structured interview studies

Setting and Participants: Family carers of relatives residing in care homes in the UK and the Netherlands were interviewed remotely.

Methods: Family carers were asked about their experiences of care home visits during the pandemic, and specifically in the Netherlands after care homes had reopened. Transcripts were analyzed in each country separately in the native language using thematic analysis, before discussing findings at multiple analysis meetings.

Results: Across 125 interviews, we developed four themes: (1) different types of contact during lockdown; (2) deterioration of resident health and well-being; (3) emotional distress of both visitors and residents; and (4) compliance to guidelines and regulations. Visiting in both the UK and the Netherlands was beneficial, if possible in the UK, yet was characterized by alternative forms of face-to-face visits which was emotionally distressing for many family carers and residents. In the Netherlands, government guidance did enable early care home visitation, while the UK was lacking any guidance leading to care homes implementing restrictions differently.

Conclusions and Implications: Early and clear guidance, as well as communication, is required in future pandemics, and in this ongoing pandemic, to enable care home visits between residents and loved ones. It is important to take learnings from this global pandemic to reimagine long-term care, highlighting the value of socializing for care home residents.

Key words: dementia, care homes, COVID-19

\section{Introduction}

Since the global COVID-19 outbreak in 2020, care homes across the world have taken restrictive measures to stem infection rates and safeguard their vulnerable older adult residents. In the UK, there

Correspondence should be addressed to: Clarissa Giebel, Department of Primary Care \& Mental Health, University of Liverpool, Liverpool, UK Email Clarissa.Giebel@liverpool.ac.uk. Received 26 Oct 2021; revised version received 06 Dec 2021; accepted 09 Dec 2021. First published online 18 January 2022. was no government guidance on how care homes should operate and how they should enable family visitation, leading care homes individually to all close down to outside visiting from February 2020 onward. Throughout the pandemic and in between waves one and two, care homes in the UK have made individual decisions of how to ease and then tighten visiting rules again. In the UK, there was a substantial lag before effective and sufficient personal protective equipment (PPE) was available for staff, residents or visitors. 
There was a significant outbreak of COVID-19 in almost 7000 English Care homes (44\%) between 9 March and 19 July 2020 with 19,286 deaths of care home residents involving COVID-19 between midmarch and mid-June 2020 (Office for National Statistics (ONS), 2020), and a further 16,355 in wave two from 1 November 2020 to mid-February 2021 (35,641 in total) in England and Wales (Nuffield Trust, 2021). In the Netherlands, both during the first and second wave, over 800 Dutch care homes had a COVID-19 outbreak (approx. 35\%). Care home residents have been most affected by COVID-19, with approximately $50 \%$ of all COVID-19 deaths during the first wave occurring within care homes, estimated at 7,400 COVID-19 deaths in care homes $(29 \%$ of total deaths in care homes) (Inzitari et al., 2020). During the first wave of COVID-19, restrictive measures in the Netherlands included a total ban for visitors to care homes. This total lockdown of care homes lasted 2 months, after which a national pilot was started to cautiously reopen care homes for visitors. In a selection of homes, one visitor per resident was allowed.

Many care home residents are aged $65+$ years and some live with dementia. Evidence is starting to emerge on how the pandemic and associated restrictions are impacting on people living with dementia in general, focusing on those residing in the community (Giebel et al., 2021; Giebel et al., 2021; Talbot and Briggs, 2021; Thyrian et al., 2020). People with dementia are found to deteriorate faster during lockdowns and restrictions in their own home, being confined to their place of residence and not receiving external face-to-face social support. This is also impacting on the mental well-being of family carers, as carers have to take on additional caring duties (Hanna et al., 2021). Evidence on the impact of the pandemic on care home settings is sparser however, with little evidence to date on how residents are faring during continued lockdowns of care homes and changing restrictions.

Verbeek et al. (2020) was the first study to report on compliance and experiences with allowing visitors back into nursing homes after a ban during the COVID-19 pandemic in the Netherlands. The study showed that in general the experiences with reopening the nursing home for visitors was very positive. Furthermore, even though there were national guidelines, there was diversity among nursing homes with regard to the use of PPE, and the arrangements of the visits. Findings from this study were mainly based on a questionnaire and interview with formal contact persons in different nursing homes. The authors of the study indicated that more research is needed into in-depth experiences of family, residents, and staff in order to investigate the impact of the visitor ban and the reopening of nursing homes. A subsample of those nursing homes was monitored during reopening using a digital questionnaire, on-site observations and in-depth interviews (Koopmans et al., 2021). While reopening appeared to be beneficial to the well-being of residents, healthcare professionals expressed concerns over increased risks of infection while acknowledging the benefits of reopening. This is supported by more research emerging on infection transmission of COVID-19 in care homes (Burton et al., 2020; Stall et al., 2020), as opposed to the experiences and psychological impacts of lack of visitations on family members and residents. However, research across the globe indicates the fine balance that needs to be struck between managing infection risks and quality of life for residents (Ayalon and Avidor, 2021; Sizoo et al., 2020; Van et al., 2020). In particular, residents and carers appear to face high levels of emotional burden due to care home closures to the outside world (Ayalon and Avidor, 2021; Van et al., 2020), while a small Delphi panel made up of 21 USA and Canadian post-acute and long-term care experts yielded five recommendations for care home visiting during the pandemic, including stringent infection control measures, enabling visits, and limited physical contact between family members and residents (Bergman et al., 2020). Thus, drawing together findings from individual countries has highlighted to date the need for safe visiting in care homes (Low et al., 2021). However, there appears to be a lack of qualitative crosscountry data comparing the experiences of different types of care home visitation during the COVID-19 pandemic so that comparisons between different countries is important to make the best use of existing knowledge to further advance care for residents during the pandemic and beyond.

The aim of this international study was to explore the experiences of family members of care home residents regarding care home visiting during the COVID-19 pandemic and compare these between the UK and the Netherlands. The Netherlands appears to have been the first country to have implemented consistent guidance for care home visitation during the pandemic and indeed has been the only country to mandate the reopening of care homes to visitors (Low et al., 2021). In contrast, there has been a lack of clear guidance on care home visitation in the UK. This provides an important perspective for exploring how care home visits were undertaken in different countries and their potential variations and similarities in affecting family members of care home residents. Despite varied degrees of vaccine rollouts across the globe, COVID-19 is going to remain an environmental infection threat for some time to come, particularly in the light of continued new virus strains developing which may 
render vaccines ineffective, access to vaccines worldwide and antivaccination movements.

\section{Methods}

\section{Participants and recruitment}

Family carers aged $18+$ years with a relative residing in a UK or Dutch care home were eligible to take part. In the UK, unpaid carers were recruited via third sector organizations, targeted emailing of care homes as part of the NIHR ENRICH network, a network of research-ready care homes, as well as via social media. At the point of data collection, there were different restrictions in place for each care home, while during November, care homes went into full lockdown. In the Netherlands, family carers who visited their family member at the beginning of the cautious reopening were informed about the study while they were receiving information on the regulations and guidelines with regard to their visit (via phone, and again right before the time of the visit). Immediately after the visit, the family carers that were willing to participate, were asked to perform a short interview (after signing an informed consent). In addition, potential participants were asked if they were willing to participate in a more in-depth phone interview 1 week after their first visit.

Ethical approval was obtained prior to study begin from the [BLINDED] ethical committee in the UK [Ref: 7626], and from the ethics committee of [BLINDED] (2020-6549) in the Netherlands.

\section{Data collection: Data and procedure}

Our data comprise of three parts - in-depth interviews from the UK were conducted between October and November 2020. Short interviews and in-depth interviews from the Netherlands were both conducted during May 2020. The short interviews were conducted immediately after the first visits since the lockdown. The in-depth interviews were performed within 1 week following the first visit. While studies in the UK and the Netherlands were conducted separately, they focused on the same topics in both countries and were thus comparable.

In the UK, topic guides for family carers and for care home staff were co-produced in a team of academics, clinicians, service providers, and unpaid carers of people living with dementia who have experience of care homes. Topic guides involved questions surrounding their experiences of visitation, how the pandemic has changed different aspects of seeing their relative with dementia in the care home, communication from the care home, as well as safety procedures and how care provision has changed for care home staff compared to before the pandemic, and how they experience residents to experience these changes. Appendix 1 includes all topic guides.

In the Netherlands, short interviews were aimed at capturing the experiences of the family carers during the lockdown and their first visit to the nursing home after the lockdown. The focus was on the impact on their well-being, and the possibility to comply with all guidelines that were in place (e.g. $1.5 \mathrm{~m}$ distance during the visit, no touching, wearing a mask, etc.). The short interviews were held for 5 consecutive days. A group of researchers was present at a nursing home during the first 2 to 3 weeks of the reopening. All nursing home residents were scheduled to receive a visitor once during the first week, where they had a visitor. Unpaid carers who were scheduled to visit the nursing home were informed about the presence of the research team and were asked to provide consent for having a short interview with one of the researchers after they visited their family member.

In addition, in-depth interviews were scheduled with participants who already took part in the short interviews. Short interviews therefore provided the first picture of the experiences, which was more extensively elaborated on in longer follow-up interviews for some family carers, with both together providing a rich picture of the nuanced experiences of carers. Furthermore, in four other care homes, visitors were asked to participate in an interview by telephone which was to be held within a few days after the visit. During this in-depth interview, participants were asked about the process of planning their visit, the communication regarding the visitation, and again, compliance and impact on wellbeing were discussed.

\section{Data analysis}

Background characteristics are described using frequency analysis in SPSS Version 25. The interviews were summarized in a brief transcript (for the short interviews) or in a structured response sheet (for the in-depth interviews). In these response sheets, (short) summaries on the response of participants were given for each of the posed questions. Next, the data were analyzed thematically within the research team using thematic analysis (Braun and Clarke, 2006). Open codes were given to sentences or paragraphs within the summaries, after which codes were grouped into overarching topics/themes. The codes and themes were discussed repeatedly in order to reach consensus on the most relevant themes with regard to the research question. 


\section{Public involvement}

Carers and people with dementia were involved in different aspects of this study in the UK. Three current and former carers were involved in all elements of this study, from conceptualization to conduct, analysis and dissemination. Public advisers, who were reimbursed for their participation, helped develop the topic guides, ensuring that the questions asked captured the important points in the lives of family members with relatives residing in care homes. There was no public involvement in the Netherlands research planning and delivery.

\section{Results}

\section{Background characteristics}

A total of 125 family carers (99 Dutch; $26 \mathrm{UK}$ ) participated in the study. Of the 99 Dutch interviews, 65 short interviews lasting between 5 and 15 minutes and 34 in-depth phone interviews lasting between 30 and 40 minutes were conducted. Table 1 provides some background demographics of the interviewed carers. In the UK, the majority of carers were female $(69.2 \%)$ and adult children of the care home residents $(61.5 \%)$. In the Dutch sample, demographics were gathered on the participants of the in-depth interviews $(n=34)$. Half of the included carers were male and half were female. The majority $(70 \%)$ of carers were the adult child of the resident.

\section{Qualitative findings}

We developed four themes using thematic analysis: (1) different types of contact during lockdown; (2) deterioration of resident health and well-being; (3) emotional distress of both visitors and residents; and (4) compliance to guidelines and regulations. Quotes for each theme and subtheme are presented in Table 2.

THEME 1: Different TyPES OF CONTACTS DURING LOCKDOWN

Very limited face-to-face contact In the UK, many carers were not allowed face-to-face visits either since the pandemic started or at different time points of the pandemic. Some were allowed to have face-toface visits in the garden, in customized pods or through windows, and a rare exception was for a carer to visit their relative in their room. Where visits were allowed, care homes had booking systems in place to manage the number of people in the homes at any time, as well as care home staff to facilitate visits with family members. Similarly, in the Netherlands, relatives indicated that during the lockdown they had no face-to-face contact with their relatives. However, the majority of respondents from both countries indicated that the nursing homes employed creative solutions to stay in contact with each other. Respondents talked about the possibility to have window visits, to talk over the phone on a regular base, sharing information via internet platforms, and to talk to their loved ones via video calling.

In the UK, some carers noted preferential visiting rights by some family members, while they themselves were not allowed to go in or only rarely. This caused some frustration, as care homes also failed to communicate why certain residents were allowed more visits than others. In the Netherlands, during the reopening of the nursing homes, each resident was allowed only one visitor. However, most respondents indicated that it was quite straightforward to decide who this person would be (as most of the time there is a "first responsible family carer").

Remote vs. face-to-face visits Both remote and the new pandemic-created face-to-face visits seemed to be of much less benefit to family members and residents as opposed to true face-to-face contacts experienced and enjoyed prior to the pandemic. In both countries, respondents indicated that visits were more beneficial than remote engagement; however, these also had their downsides. Garden visits, for example, were weather-dependant, and if the weather was not suitable, window visits were conducted instead, where care homes enabled these. There was an issue for some residents who were not living on the ground floor, being excluded from window visits and causing further distress to the new forms of alternative visiting forms. Many carers shared how their relative with dementia was unable to understand socially distanced face-to-face visits or hear their relative from a distance, highlighting how alternative visiting options could not replace real face-to-face contacts.

Where remote contact was enabled digitally, such as via skype, there was rarely any privacy, as care home staff had to support the resident in using a phone or tablet, and often with limited understanding from the resident. Carers expressed concerns about being unable to have a private conversation with their relative. Pod visits similarly offered little privacy, highlighting overall how different types of COVID-19 visits have changed the dynamics, compared to visits in the resident's room for example.

Some carers noted that the types of visits that were allowed would not have been taken up by the residents if it was pre-pandemic. For example, one UK carer explained that her husband would never have sat in the garden, so having a pandemic garden 
Table 1. Participant characteristics of the Dutch in-depth interview and the UK sample

\begin{tabular}{|c|c|c|}
\hline & $\begin{array}{l}\text { DUTCH SAMPLE (IN-DEPTH INTERVIEWS) } \\
\qquad \mathrm{N}=34\end{array}$ & $\begin{array}{l}\text { UK SAMPLE } \\
\qquad \mathrm{N}=26\end{array}$ \\
\hline \multicolumn{3}{|l|}{$\mathbf{N}(\%)$} \\
\hline \multicolumn{3}{|l|}{ Gender } \\
\hline Male & $17(50 \%)$ & $8(30.8 \%)$ \\
\hline Female & $17(50 \%)$ & $18(69.2 \%)$ \\
\hline \multicolumn{3}{|l|}{ Relationship with resident } \\
\hline Daughter/son (in law) & $24(70.6 \%)$ & $16(61.5 \%)$ \\
\hline Spouse & $6(17.6 \%)$ & $10(38.4 \%)$ \\
\hline Other & $4(11.7 \%)$ & \\
\hline \multicolumn{3}{|l|}{ Mean (+/-SD) [range] } \\
\hline Age (mean) & $58(+/-13)[25-84]$ & $63(+/-9)[42-89]$ \\
\hline Normal number of visits per week (mean) & $3.7($ Range $=1-7), \mathrm{SD}=2,1$ & $\mathrm{n} / \mathrm{a}$ \\
\hline $\begin{array}{l}\text { Length of stay of resident in nursing home in years } \\
\text { (mean) }\end{array}$ & $3($ Range $=0,3-13), S D=3,3$ & $\mathrm{n} / \mathrm{a}$ \\
\hline
\end{tabular}

visits would be of no use and benefit. Equally, pod visits or window visits would never have been used pre-pandemic and outside of an infection-controlled environment.

\section{Missing the personal touch}

While carers benefited to some extent from the socially distanced face-to-face visits, carers also expressed their upset about needing to hold their relative's hand to feel close to them. Missing the personal human touch between family members and residents was also expressed as an issue where care home residents did not comprehend window visits and for example got agitated due to the inability to be close to their family member. Although in the Netherlands everyone was happy to be able to visit again, due to the guidelines of keeping distance, no touching, and the wearing of face masks, a visit was sometimes experienced as disappointing, difficult, or nervous.

\section{THEME 2: Deterioration of RESIDENT} HEALTH AND WELL-BEING

Noticing faster deterioration Carers in both countries noticed an increased deterioration in symptoms and the condition of their relatives with dementia during the pandemic and since lockdowns and other restrictions had commenced. This not only included the residents' cognitive and physical symptoms, but also their well-being. Where some residents had been active before the pandemic, since then carers were complaining and concerned about the deteriorations in mobility within often relatively short periods of time, both in the Netherlands and the UK.
THEME 3: EMOTIONAL Distress OF Both VISITORS AND RESIDENTS

Concern over relative Many family members were concerned over their relative's well-being and their relationship with them. The increased periods of being unable to visit their relative worried carers, especially where they received little communication from the care homes about their well-being. In effect, care home staff took on an unplanned new role of family, caring for the residents at all times and not allowing their real family to enter the care homes. This was heightened by the fact that all UK residents and many Dutch residents were living with dementia, and thus more likely to forget their own family members by being unable to see them on a regular basis.

Worry about transmitting virus Visiting their relative posed mixed feelings to visitors. Many enjoyed visiting their relative but many were also scared of the risks posed by reopening care homes. Reopening care homes could result in increased risks of virus transmission, without sufficient testing and other restrictions in place, which left some carers accepting that care homes should stay closed in the UK. Others however, in the UK, were frustrated with the lack of visiting and were desperate for care homes to open their doors again to properly connect and communicate with their relative again.

Emotional response from visitors and residents/intensity of emotions Visitors indicated that it was good to see each other after a long time. However, Dutch and UK experiences seemed to differ slightly in terms of emotional impact on both carers and residents. In the Netherlands, most residents were happy and 
Table 2. Qualitative quotes by themes and subthemes

THEME QUOTES

\section{THEME 1: Different types of contacts during lockdown}

Very limited face-to-face contact

"We have no communication with them whatsoever in terms of the changes. They might in the summer when they decided garden visits could happen that was just by chance and one of them you know the activities coordinator telling me there's been no official written information or anything about any anything." UK ID21, female carer

"They have purchased a phone for my mother, however she does not understand how it works. Therefore, I now call to the ward every day, and the staff then makes sure I can video-chat with her. So now me and my brothers call her every day." Dutch ID0512E3, daughter of resident

"I didn't get told she'd had a fall; my brother rang me which is what I didn't want. So after it I did say to the home I need to be contacted I have asked you erm they did apologise for that erm but I went in the other week and they've changed her GP and she said you got the letter and I said no I didn't get a letter." UK ID10, Female carer, Daughter

Remote vs. face-to-face visits

"They made like French doors out to the outside where there had been a window and they built a room with a glass partition with a door in it across the middle of the room and most importantly it had a very good microphone and speaker system in it like a conference phone and I visited mum in there once but and sadly for me and for mum it didn't work. Because she couldn't, she was obviously failing as well she didn't realise I was on the other side of the glass, I think she sort of looked at she could only see the glass but she couldn't appreciate that I was on the other side." UK ID12, male carer, son

"I did a window visit once, however my mother responded poorly to that, as it was a scary situation for her, as we could not touch each other for instance." Dutch ID0511J3, daughter of resident

"I have to talk to her in the presence of the carer which I don't mind particularly but obviously it's not private or personal and it's dependent on what sort on what sort of mood she's in whether she's talking to you know my dad and my grandmother and everybody or whether she's asleep. But err recently she's been, if I get the same carer she really really relates to one carer so I've arranged that I only Skype now when she's working and then it works quite well. I mean we sing songs together and I can show her round my house and go into the garden and things." UK ID20, family carer

"We do video calls but it isn't the same he doesn't quite understand well he doesn't understand full stop what's happening. I suppose in some ways it might be a good thing to have dementia because you cannot figure out what's going on in the world." UK ID01, female carer

"They're still saying well you can do garden visits and [person] had said well who's going to want to sit in the garden in October anyway. [Person with dementia] if he were able to say anything even in his normal stay at home he wouldn't have gone and sat in the garden in this weather." UK ID02, Female carer, Spouse

Missing the personal touch

"It's absolutely urgent that essential carers get into homes but it need to be managed and it can be managed. I want to, I offered months ago to have a test to have regular tests to wear the aprons and the gloves but sanitize my hands, wear a visor not a mask and hold [relative's] hand because if I don't hold his hand then I might as well not be there." UK ID02, female carer, spouse

'Normally I always hug and kiss her when I visit, and that's very different now. I would have preferred to be able to visit in her own apartment, and to be able to actually hug her, however that's not possible at the moment." Dutch ID NCVC03, son of resident 


THEME $\quad$ QUOTES

THEME 2: Deterioration of resident health and well-being

Noticing faster deterioration "I visited my father and I noticed a strong deterioration compared to 8 weeks ago. My father was just lying in bed the whole time, he was not really present anymore, and very hard to talk to, to get in contact with. This was a strong contrast with 8 weeks ago, then he was much more active, and easy to connect with. Also, I noticed he had very skinny arms and legs, and I heard that he fell out of bed a lot." Dutch ID0513H3, son of resident

THEME 3: Emotional distress of both visitors and residents

Concern over relative

Worry about transmitting virus

Emotional response from visitors and residents/Intensity of emotions

THEME 4: Compliance to guidelines and Respecting the importance of guidelines

Difficulties with adhering to guidelines
"It's quite upsetting knowing the way that it's like he's got a new family and because he lives with them all the time he can forget me obviously." UK ID01, female carer

"If I have any queries it's with how government have handled the whole thing really and I think I do think, well I utterly understand that they can't let visitors in because you know danger of death, I completely understand that, at the same time I do think we're being held hostage because they [messed] up at the beginning." UK ID23, Female carer, daughter

"She gives a bit of a blank look now at me. It's really hard, it's almost like she relies on the staff now which is nice in one way. Because they're with her $24 / 7$ but it's like she got upset and hugs them and I'm sat across the glass thinking [wanting to be] holding her hand and me giving her the hug." UK ID10, female carer, daughter

\section{regulations}

"It was quite easy to follow all the rules. It's fine like this. Luckily, my father isn't really much of a hugger, so keeping distance is also easy." Dutch ID $0512 \mathrm{E} 7$, son of resident

"I think he's probably getting used to people wearing PPE all the time, particularly in the hospital so he was fairly laid back about it yesterday and I was able to hold his hand because I was wearing gloves as well so that was good. Because that touch is so important." UK ID28, family carer

"Not touching belongings is impossible. I brought clean laundry for my mother and put those in the closet. I also wanted to make coffee for me and my mother, which is something that you normally do when visiting. The staff did not want me to do that, but allowed it anyway. It is also strange that I was not allowed to bring strawberries that I prepared at home. After all, I did disinfect my hands an extra time." Dutch (ID0512E3)

"I thought how can you leave someone who's got, who can't hear and there's face masks on so can't lipread and has not mental capacity to quite understand and grasp and that's, I felt like she was just left you know and it was like oh my god I wouldn't even do that to my dog." UK ID17, female carer, daughter comfortable during visits, whereas a few were sleepy/ absent or confused. Face-to-face contact had added value, but relatives also mentioned that visits were different and less enjoyable than before due to the lack of physical contact and not being allowed to walk outside. Some residents got agitated not understanding why they could not touch their relative or why they were behind a window or screen. These negative experiences were reflected in many UK carers, with residents being agitated for a lack of understanding the physical distance and screens between them and their loved ones, leading to emotional upset in many carers.
THEME 4: Compliance to Guidelines And REGULATIONS

Respecting the importance of guidelines In the Netherlands, all respondents indicated to understand the importance to adhere to all the guidelines. The initial response of most participants was that it was easy to adhere to the guidelines. Similarly, UK carers expressed it was important to adhere to the guidelines. However, where the resident was in the end of life stages, carers were allowed to hold their relative's hand with restrictions eased surrounding physical distancing. 
Difficulties with adhering to guidelines While family carers mostly respected the guidelines, some family members also expressed how difficult it was to adhere to them in both countries. They missed the personal touch and struggled keeping a physical distance from their relative when on a face-to-face visit. It seemed unnatural to keep a distance from their relative who they have not seen for a long time. Others also mentioned the difficulties of wearing masks when on visits, and the difficulties in engaging with their relative this way, as the person with dementia cannot see facial features and the family member smiling for example, and also may struggle recognizing the family member. This was also raised by a UK family carer in light of staff wearing PPE in the care homes which appeared to cause difficulties and in the carer's eyes, a care neglect.

\section{Discussion}

This is one of the first studies to explore the impact of the pandemic on visiting care home residents and comparing the experiences across two cultural settings. While remote and the new normal alternative types of face-to-face visits were available, they were unable to replace genuine face-to-face visits in both countries. The difference between countries was that the Netherlands had quickly implemented blanket guidance on care home visitation, enabling a faster and smoother transition to reuniting family members with residents again.

Removing any face-to-face contact between family members and residents and creating a care home contact bubble has detrimental effects on the wellbeing of both parties involved. During the pandemic, alternative face-to-face visits such as window, pod, or garden visits have become the norm, where they were available and enabled by care home staff. In addition, remote digital connections between family members and residents also have become the new norm, reflecting what has occurred in community settings for people with dementia and carers trying to engage with support services (Arighi et al., 2021; Giebel et al., 2021).

While these were a lifeline to stay in contact with relatives to some degree, many family members faced difficulties in engaging with different face-to-face visits in the UK. This was because of a lack of clear guidance for care homes, whereas in the Netherlands, guidance was released and implemented early on in the pandemic in May 2020. Thus, comparing the data from these two countries with very different levels of government input as to how visiting should operate, findings indicate how Dutch family members have benefited to a greater degree from these alternative visits, leaving many UK family members excluded from care homes for long periods of time.
While guidelines were helpful in the Netherlands in enabling visits relatively early on in the course of the pandemic, family members in both countries expressed how they respected and understood the general public health measures (such as social distancing, wearing PPE). However, they also raised the difficulties they experienced by not holding a relative's hand on a visit especially for those with sensory deficits. Considering the emotional turmoil experienced by lack of contact between residents and family members, and the restricted nature of contact. Findings indicate the significance of social contact and their impact on mental well-being, as evidenced outside the care home sector during the pandemic and in pre-pandemic times (DomenechAbella et al., 2019; Cations et al., 2021; Cohen et al., 2020). This is also captured in a recent international report on the care home visiting, which provides five major recommendations which are corroborated by our findings: avoiding blanket visitor bans; safe alternative face-to-face visits; essential partner status for family carers; government support for implementing safe visiting; and ensuring the human rights of residents are met, not depriving them of their basic rights of seeing friends and family (Low et al., 2021). Restrictions in care homes thus need to be mindful of the individual resident's needs, rather than a blanket ban on family members per se. If not, care homes indeed risk, and have risked, human rights violations, also in light of quarantining residents with dementia against their will (and understanding) (Iaboni et al., 2020) so that Low's et al.'s (Low et al., 2021) recommendations should be implemented across care homes, particularly in countries where governmental guidance is missing.

This lack of government guidance has left care homes across the UK, and globally, without clear guidance about how to manage infection risks as well as to manage the residents' well-being or justify the choices they make in this regard. There was no clear guidance on how to provide care safely to residents, and care homes had to come up with their own strategies of how to deliver care, but also how to enable or minimize outside visits (from family members and friends) in order to reduce the spread of the virus. In addition, procuring PPE remains a significant issue for many care homes and social care staff more broadly (Carter, 2020; McGarry et al., 2020). Without any guidance, and staff more likely to work across different care homes, infection risks and COVID-19 outbreaks are higher (Ladhani et al., 2020). Thus, a key message from this pandemic and from this study is the importance of clear communication to care homes, and family members, in case of future pandemics or future waves of COVID-19.

While data were collected prior to vaccination rollouts, findings have implications for vaccination ready 
care homes as well, as well as for new COVID-19 waves with new variants which are not targeted by current vaccines. In light of a large vaccination roll-out in the UK since early December 2020, from 8 March and the 29 March 2021, one and two essential visitors, respectively, have been allowed into the care homes again while strictly adhering to public health guidance. Although this guidance was released, it was not implemented in all care homes in March, with factors of low uptake of vaccination in care home staff likely also affecting family visitation (Giebel et al., ). In contrast, in the Netherlands, it is now illegal to impose a full lockdown on care homes, something that has not been enshrined in law in the UK, yet. However, there is no evidence to date on how these guidelines are implemented in practice, and future research needs to explore the effects of vaccinations and new visitation rules on residents and family members.

While this study benefits from a cross-country exploration of the topic and thus wider representativeness of the findings, it is to be noted that this study only focused on family carers. These also shared an insight on how residents were faring during those visits, but findings are restricted to their perspectives. Considering the barriers of getting ethical approval to conduct interviews with residents in the UK, linked with the inability to go into care homes to collect data, obtaining the views from family carers are a suitable avenue of obtaining data. Family carers completing a short interview in the Netherlands were all from one care home, thereby reducing the wider representativeness of the short interviews while providing a rich picture of the different experiences of family carers. Longer interviews were conducted across different sites. Another limitation is that the topic guides in both countries were not the same, yet asked similar questions and thus focused on the same topic, making it possible to compare the data from both studies.

\section{Conclusions}

Care homes require clear guidance to be able to deal with infection control effectively and manage new ways of care delivery. While new forms of visiting, such as window and pod visits, as well as remote digital connections, do not provide the same level of connection between family members and residents as pre-pandemic face-to-face visits do, enabling these well and frequently to all family members and residents can help reduce some of the emotional burden felt by lack of contact. Considering patchy vaccination roll out worldwide, and new variants found regularly which may impede the benefits of existing vaccinations, we can take important learnings from this international study and provide strong recommendations for improved, early, and clear guidance for care delivery and visitation, and the enablement of (alternative) face-to-face visits as much as possible. The early implementation of care home visitation in the Netherlands has highlighted the benefits of care home visitation, if properly supported through information and support for visitors, staff, and residents, which should be provided in future outbreaks in care homes across the world.

\section{Acknowledgments}

We wish to thank all the participants who shared their experiences as part of this study, highlighting the issues surrounding care in the time of COVID- 19 .

\section{Conflicts of interest}

None.

\section{Funding}

This study was funded by the Geoffrey and Pauline Martin Trust, with funding awarded to the principal investigator. This is also independent research funded by the National Institute for Health Research Applied Research Collaboration North West Coast (ARC NWC). The views expressed in this publication are those of the author(s) and not necessarily those of the National Institute for Health Research or the Department of Health and Social Care. The Dutch study was funded by the Dutch Ministry of Health, Welfare, and Sports. The research was coordinated by the University networks of Radboud University Medical Centre and Maastricht University; data have been collected by six University networks in the care for older people.

\section{Description of author roles}

CG conceptualized and led the study, CG, MG, and PM designed the topic guide jointly in the UK and discussed the findings jointly, BdB, HV, DG, and AS designed the topic guide in the Netherlands, BdB analyzed the data, CG and MG analyzed the data in the UK, CG and BdB compared the findings across the countries, CG wrote the manuscript, and all co-authors approved drafts of the manuscript and the final version.

\section{Supplementary material}

To view supplementary material for this article, please visit https://doi.org/10.1017/S1041610221002799 


\section{References}

Arighi, A. et al. (2021). Facing the digital divide into a dementia clinic during COVID-19 pandemic: caregiver age matters. Neurological Sciences, 42, 1247-1251.

Ayalon, L and Avidor, S. (2021). 'We have become prisoners of our own age': from a continuing care retirement community to a total institution in the midst of the COVID-19 outbreak. Age E Ageing. doi: 10.1093/ageing/afab013.

Bergman, C. et al. (2020). Recommendations for welcoming back nursing home visitors during the COVID-19 pandemic: Results of a Delphi panel. $\mathcal{F A M D A}, 21,1759-1766$.

Braun, V. and Clarke, V. (2006). Using thematic analysis in psychology. Qualitative Research in Psychology, 3, 77-101.

Burton, J. K. et al. (2020). Evolution and effects of COVID19 outbreaks in care homes: a population analysis in 189 care homes in one geographical region of the UK. The LANCET Healthy Longevity, 1, e21-e31.

Carter, R. (2020). COVID-19: The support UK care homes need to survive. $B M \mathcal{F}, 369, \mathrm{~m} 1858$.

Cations, M., Day, S., Laver, K., Withall, A. and Draper, B. (2021). People with young-onset dementia and their families experience distinctive impacts of the COVID-19 pandemic and associated restrictions. International Psychogeriatrics, 33, 839-841.

Cohen, G., Russo, M. J., Campos, J. A. and Allegri, R. F. (2020). Living with dementia: increased level of caregiver stress in times of COVID-19. International Psychogeriatrics, 32, 1377-1381.

Domenech-Abella, J., Mundo, J., Haro, J. M. and Rubio-Valera, M. (2019). Anxiety, depression, loneliness and social network in the elderly: Longitudinal associations from The Irish Longitudinal Study on Ageing (TILDA). Fournal of Affective Disorders, 246, 82-88.

Giebel, C. et al. (2021). COVID-19-related social support service closures and mental well-being in older adults and those affected by dementia: a UK longitudinal survey. BMF Open, 11, e045889. doi: 10.1136/bmjopen-2020045889 .

Giebel, C. et al. (2021). Impact of COVID-19 related social support service closures on people with dementia and unpaid carers: a qualitative study. Aging $\mathcal{E}$ Mental Health. doi: 10.1080/13607863.2020.1822292.

Giebel, C. et al. (2021). Navigating the new normal: accessing community and institutionalized care for dementia during COVID-19. Aging $\mathcal{E}$ Mental Health. doi: 10.1080/13607863.2021.1914545.

Giebel, C. et al. Are we allowed to visit now? Concerns and issues surrounding vaccination and infection risks in UK care homes during COVID-19. Age E Ageing. doi: 10 .1093/ageing/afab229.

Hanna, K. et al. (2021). Emotional and mental wellbeing following COVID-19 public health measures on people living with dementia and carers. Fournal of Geriatric Psychiatry and Neurology. doi: 10.1177/ 2F0891988721996816.

Iaboni, A. et al. (2020). Achieving safe, effective, and compassionate quarantine or isolation of older adults with dementia in nursing homes. American fournal of Geriatric Psychiatry, 28, 835-838.
Inzitari, M. et al. (2020). Nursing homes and long-term care after COVID-19: a new era? Fournal of Nutrition, Health $\mathcal{F}$ Aging, 24, 1042-1046.

Koopmans, R. C. T., et al. (2021). Reopening the doors of Dutch nursing homes during the COVID-19 crisis: results of an in-depth monitoring. International Psychogeriatrics, 34, 391-398. doi: 10.1017/S1041610221000296.

Ladhani, S. N. et al. (2020). Increased risk of SARS-CoV-2 infection in staff working across different care homes: enhanced COVID-19 outbreak investigations in London care homes. Fournal of Infection, 81, 621-624.

Low, L. F. et al. (2021). Safe visiting is essential for nursing home residents during the COVID-19 pandemic: An international perspective. $\mathcal{F A M D} A$. doi: $10.1016 /$ j.jamda 2021.02.020.

Low, L.-F. et al. Safe visiting at care homes during COVID19: A review of international guidelines, emerging practices during the COVID-19 pandemic. International Long Term Care Policy Network 2021. Available at: https:// ltccovid.org/wp-content/uploads/2021/01/Care-homevisiting-policies-international-report-19-January-2021-1.pdf

McGarry, B. E., Grabowski, D. C. and Barnett, M. L. (2020). Severe staffing and personal protective equipment shortages faced by nursing homes during the COVID-19 pandemic. Health Affairs, 39. doi: 10.1377/hlthaff.2020 .01269

Nuffield Trust. (2021). COVID-19 and the deaths of care home residents. London, England: Nuffield Trust. Available at: https://www.nuffieldtrust.org.uk/news-item/covid-19and-the-deaths-of-care-home-residents; last accessed 28 June 2021.

Office for National Statistics (ONS) (2020). Impact of coronavirus in care homes in England: 26 May to 19 fune 2020. London, England: ONS.

Sizoo, E. M., Monnier, A. A., Bloemen, M., Hertogh, C. M. P. M. and Smalbrugge, M. (2020). Dilemmas with restrictive visiting policies in Dutch nursing homes during the COVID-19 pandemic: A qualitative analysis of an openended questionnaire with elderly care physicians. $\mathcal{F} A M D A$, 21, 1774-1781.

Stall, N. M., Jones, A., Brown, K. A., Rochon, P. A. and Costa, A. P. (2020) For-profit long-term care homes and the risk of COVID-19 outbreaks and resident deaths. Canadian Medical Association fournal, 192, E964-E955.

Talbot, C. V. and Briggs, P. (2021). 'Getting back to normality seems as big of a step as going into lockdown': the impact of the COVID-19 pandemic on people with early to middle stage dementia. Age E Ageing, 50, 657-663.

Thyrian, J. R. et al. (2020). The situation of elderly with cognitive impairment living at home during lockdown in the Corona-pandemic in Germany. BMC Geriatrics, 20, 540. doi: 10.1186/s12877-020-01957-2.

Van der Roest, H. G. et al. (2020) The impact of COVID-19 measures on well-being of older long-term care facility residents in the Netherlands. $\mathcal{F} A M D A, 21$, 1569-1570.

Verbeek, H. et al. (2020). Allowing visitors back in the nursing home during the COVID-19 crisis: a Dutch national study into first experiences and impact on well-being. $\mathcal{F} A M D A, 21,900-904$. 